\title{
Biometría testicular y características seminales en felinos neotropicales (Carnivora: Felidae) sometidos a cateterismo uretral
}

\author{
Mónica Madrigal-Valverde ${ }^{1,2 *}$, Rodrigo F. Bittencourt ${ }^{1}$, Antonio de Lisboa Ribeiro Filho ${ }^{1}$,
} Maicon P. Lents ${ }^{1}$, Milena C. de Azevedo ${ }^{1}$, Renata O. Barreto ${ }^{1}$, Nayara A. Teixeira ${ }^{1}$, Paulo M. S. Mattos ${ }^{4,5}$, Vitor P. Curvelo ${ }^{6}$, Marta C. Gomes ${ }^{6}$, Vínicius Dantas ${ }^{6}$, Gediendson Ribeiro de Araujo ${ }^{7}$ \& Anthony Valverde-Abarca ${ }^{2}$

1. Escola de Medicina Veterinária e Zootecnia, Universidade Federal da Bahia, CEP 40170-110 Salvador, Bahía, Brasil; madrigalvalverdem@gmail.com,rfb@ufba.br, alisboa@ufba.br, maicon_pl@hotmail.com,renata.obrt@hotmail.com, naay_aquino@hotmail.com

2. Instituto Tecnológico de Costa Rica. Escuela de Agronomía. Campus Tecnológico Local San Carlos, Apto. Postal 223-21001 Alajuela, Costa Rica; mmadrigal@itcr.ac.cr, anvalverde@itcr.ac.cr

3. Hospital Veterinario Renato R. de Madeiros Neto, CEP 40170-110, Salvador, Bahía, Brasil; milenacastro92@hotmail.com

4. Instituto Gonçalo Moniz, Fundação Osvaldo Cruz, CEP 40296-710, Salvador, Bahía, Brasil; os.mattos@outlook.com

5. Facultade de Medicina da Bahia, Universidade Federal da Bahia, CEP 40110905 Salvador, Bahía, Brasil; os.mattos@outlook.com

6. Parque Zoobotânico Getúlio Vargas, CEP 40170-110, Salvador, Bahía, Brasil; vitor.curvelo@inema.ba.gov.br, marta.gomes@inema.ba.gov.br, vinicius.dantas@inema.ba.gov.br

7. Biosciences Institute, Federal Univesity of Mato Grosso do Sul, 79074460 Campo Grande, MS, Brazil, gediendson@gmail.com

\section{Recibido 18-II-2019. Corregido 23-V-2019. Aceptado 29-VII-2019.}

\begin{abstract}
Testicular biometry and seminal traits in neotropical cats (Carnivora: Felidae) by uretral catheterization. Introduction: The urethral catheterization associated with the application of alfa- 2 adrenergic agonist drug is a recent development and application technique for the collection of feline semen, with advantages over electro-ejaculation harvesting and epididymal harvesting. Objetives: Seminal collection in neotropical felines using the urethral catheterization technique. Determine the testicular and seminal parameters of samples collected by the urethral cataterization technique. Methods: The technique has been tested on Panthera onca and others small cats. In this study, individuals of Panthera onca, Puma yagouaroundi and Leopardus wiedii were used in captivity at Getulio Vargas Zoobotanical Park, Salvador, Bahia, Brazil. Medetomidine hydrochloride $(0.1 \mathrm{mg} / \mathrm{kg})$ and ketamine $(5.0 \mathrm{mg} / \mathrm{kg})$ were used as sedatives, the semen was collected by urethral catheterization. In the process were checked the testicular biometry was performed with percutaner and ultrasonography technique. The fresh samples were evaluated for conventional microscopy in the parameters of volume, sperm vigor, total motility, progressive motility, structural and functional integrity of the plasmatic membrane and sperm morphology. The samples were subjected to a freeze - thaw process using three different extenders. The thawed samples were tested using conventional microscopy, automated system analysis and flow cytometry. Results: In this study, the urethral harvest technique was successfully used in the three species. In the literature used don't have report about seminal parameters in P. yagouaroundi and urethral seminal collection in L. wiedii. The data presented describe the spermatic morphocinetics of the included species in a pioneer study. There were no differences $(\mathrm{P}>0.05)$ in the seminal quality evaluating the extender, nor differences in the integrity of pre
\end{abstract}

Madrigal-Valverde, M., Bittencourt, R. F., de Lisboa Ribeiro Filho, A., Lents, M. P., de Azevedo, M. C., Barreto, R. O., Teixeira, N. A., Mattos, P. M. S., Curvelo, V. P., Gomes, M. C., Dantas, V., Ribeiro de Araujo, G., \& Valverde-Abarca, A. (2019). Biometría testicular y características seminales en felinos neotropicales (Carnivora: Felidae) sometidos a cateterismo uretral. Revista de Biología Tropical, 67(4), 975-988. 
and post-freeze membranes of the samples. Conclusion: The urethral catheterization associated with the application of alfa- 2 adrenergic agonist drug it's an efficient technique for semen collection in neotropical cats. The establishment of a potential association of physical characteristics with seminal quality is extremely important information on the applicability of reproductive traits in assisted reproduction programs.

Key words: semen colection; Panthera onca; Leopardus wiedii; Puma yagouaroundi; CASA; flow citometry.

Las especies de felinos de incidencia en Brasil, se encuentran en peligro o potencialmente en peligro de extinción (CITES, 2017). Considerando que los felinos silvestres generalmente tienen una esperanza de vida corta, un bajo número de crías (entre 1 a 4 crías/parto) y la aparición temprana de la senescencia reproductiva (7-10 años de edad), el mantenimiento actual de las poblaciones genéticamente viables en cautiverio es muy difícil, sin embargo prioritaria (Moreira, 2017).

La reproducción en cautiverio es una de las herramientas para la conservación ex situ de los felinos silvestres, que, en la mayoría de los casos, están amenazadas de extinción. Se han realizado esfuerzos en varios frentes para evitar la desaparición de estos carnívoros en todo el mundo (Micheletti, Cubas, \& Moraes, 2012; Pukazhenthi \& Wildt, 2004). La información de la fisiología reproductiva básica es esencial para el desarrollo y aplicación de sistemas de reproducción asistida en cautiverio, técnicas tales como la inducción de la ovulación y los bancos de germoplasma (Swanson, Roth, Citinc, Kenny, \& Wildt, 1996).Para alcanzar esto es necesario que se desarrollen estudios sistemáticos (Moreira, 2017). Es de esta forma, que se resalta la recolecta de semen como una técnica fundamental en cualquier intento de aplicar la reproducción asistida de felinos silvestres.

Debido a las dificultades y los riesgos involucrados en el manejo de los animales silvestres, la técnica de electroeyaculación ha sido el método de elección para la recolecta seminal. No obstante, con las dificultades y los requisitos para utilizar este método, se han desarrollado otras metodologías de recolecta, tales como el cateterismo uretral (Zambelli, Prati, Cunto, Iacono, \& Merlo, 2008; Araujo, et al., 2018). Según Zambelli et al. (2008) y Araujo et al. (2018) mejora la recolecta espermática en los programas de reproducción asistida en los felinos silvestres. Sin embargo, para un mayor avance en la reproducción asistida de felinos silvestres es necesario ampliar los conocimientos sobre los aspectos reproductivos básicos de éstos, así como priorizar el estudio de las técnicas utilizadas en los programas de reproducción asistida con el fin de superar las dificultades y limitaciones de cada una de estas técnicas (Micheletti et al., 2012).

De esta forma, los objetivos de este estudio fueron: a) Recolectar semen de los felinos silvestres de distribución en el estado de Bahía, mantenidos en cautiverio, utilizando la técnica de cateterismo uretral, b) Evaluar el comportamiento de diferentes crioprotectores en el proceso de criopreservación seminal de muestras de felinos brasileños, c) Evaluar las muestras seminales posterior a la criopreservación por microscopía convencional, microscopía computarizada y citometría de flujo, d) Contribuir al ámbito científico con informaciones sobre parámetros seminales y cateterismo uretral en P. yagouaroundi y L.wiedii.

\section{MATERIALES Y MÉTODOS}

Las etapas experimentales de este estudio siguieron las normas aprobadas por la Comisión de Ética de Uso Animal de la Escuela de Medicina Veterinaria y Zootecnia de la Universidad Federal de Bahía (CEUA EMEVZUFBA: 10/2016) y el permiso del Instituto 
Chico Mendes para la Conservación de la Biodiversidad (ICMBio SISBIO No 47822).

Localización del estudio: El estudio fue realizado en la ciudad de Salvador, estado de Bahia, Brasil, situada en las coordenadas geográficas latitud $12^{\circ} 58^{\prime} 16^{\prime \prime} \mathrm{S} \& 38^{\circ} 30^{\prime} 39^{\prime \prime} \mathrm{W}$, a una altitud de 8 m.s.n.m., con una temperatura promedio de $24{ }^{\circ} \mathrm{C}$ a $26{ }^{\circ} \mathrm{C}$ y una precipitación total anual de 1200-1400 mm. Los felinos provenían del parque zoobotánico Getúlio Vargas propiedad del gobierno de la República Federativa de Brasil. Las etapas de laboratorio fueron realizadas en el Sector de Reproducción Animal y Obstetrícia Veterinaria (RAOV-HOSPMEV / UFBA) del Hospital Veterinario Renato Rodenburg de Medeiros Neto (HOSPMEV/ UFBA), ubicado en el campus universitario Ondina, de la Universidad Federal de Bahía.

Animales: Fueron utilizados tres $(\mathrm{n}=3)$ machos adultos de las siguientes especies: un caucel (Leopardus wiedii), un león breñero (Puma yagouaroundi) y un jaguar (Panthera onca). Los individuos habían alcanzado la madurez sexual y no presentaban desnutrición y/o condiciones que indicaran problemas de salud.

Anestesia y captura: Cada animal fue sometido a una recolecta de semen. Antes de la realización de la recolecta, los animales fueron contenidos químicamente, con el uso de los fármacos en las siguientes dosis, clorhidrato de medetomidina $(0.1 \mathrm{mg} / \mathrm{kg})$ y ketamina $(5.0$ $\mathrm{mg} / \mathrm{kg}$ ) administrados por vía intramuscular. El anestésico fue administrado por dardos preparados y proyectados por cerbatana. Fue esperada la acción del fármaco anestésico en el recinto del animal. Una vez alcanzado el estado anestésico adecuado, fue cubierta la cabeza con un paño de tela y el animal fue trasladado a un área preparada para realizar el proceso de recolecta seminal. Se tomaron los signos vitales de frecuencia cardiaca, respiratoria, saturación de oxígeno y presión arterial promedio, diastólica, sistólica y temperatura corporal por medio de un monitor multiparamétrico.
El examen físico de los animales: Los animales se evaluaron clínicamente y se recogieron muestras de sangre, orina y heces para comprobar la salud de los individuos con el fin de evitar la evaluación del semen de animales que presentaran condiciones de salud que comprometieran la calidad del esperma. Los testículos, pene y prepucio de los animales fueron inspeccionados en cuanto a la presencia de ectoparásitos y lesiones. Se verificó la presencia de espículas peneanas en el caso del $P$. onca y P. yagouaroundi. Posteriormente, los testículos se palparon para la evaluación de la consistencia (suave, fibroelástica, dura) y la movilidad del testículo dentro del escroto.

Biometría y la ecografía: Para realizar la biometría testicular, los testículos fueron medidos utilizando un pie de rey digital (LT-4237-000 Electronic Digital Caliper). Se midieron las dimensiones de la longitud, ancho y grosor del testículo, así como el doble pliegue de piel del escroto (Azevedo et al., 2010). La evaluación ecográfica permitió la verificación de la ausencia de condiciones internas del testículo que pudieran influir en la salud y la aptitud reproductiva de los animales. Se utilizó un equipo ultrasonográfico Mylab Five (Grupo Esaote, Barcelona, España) con un transductor lineal en modo trapezoidal a una frecuencia de $5 \mathrm{MHz}$ para determinar las medidas de longitud y anchura testicular.

Parámetros testiculares: A partir de los datos obtenidos vía pie de rey (largo, ancho y grosor) fue estimada el área de cada testículo $\left(A=\pi r^{2}\right)$ en donde $r^{2}$ se obtiene multiplicando la mitad de la longitud por la mitad de la anchura testicular. El volumen de los testículos (Método 1) fue estimado mediante la fórmula del volumen del elipse y se calculó con base en la fórmula de volumen del elipse $\left(\mathrm{V}=\frac{4}{3} \pi \mathrm{r}^{3}\right)$ donde la operación se obtiene multiplicando la mitad del ancho * (multiplicado por) la mitad del ancho * la mitad del grosor del testículo. A cada medida se le sustrajo el grosor de la piel del escroto. Con el volumen obtenido fue calculado el peso testicular, 
testicular utilizando la densidad del testículo del mamífero de $1.046 \mathrm{~g} / \mathrm{mL}$ (Johnson, Petty, $\&$ Neaves, 1981). Con este peso testicular y el peso total del animal fue calculado el índice de masa localizado en la gónada del animal llamado índice Gonadosomático (IG).

Con las imágenes que fueron capturadas por la ecografía de cada testículo (Método 2) fue estimada el área testicular, según la fórmula de área antes expuesta y el volumen testicular fue $\left(\mathrm{V}=\frac{4}{3} \pi \mathrm{A}^{2} * \mathrm{~B}\right)$ : donde $\mathrm{A}$ es la mitad de la anchura y B es la mitad de la longitud (Howard, Brown, Bush, \& Wildt, 1990; Villaverde et al., 2014). Adicionalmente, con las mediciones obtenidas vía ecografía, se estimaron el peso testicular y el IG como se mencionó anteriormente.

Recolecta de semen: Entre 15 y $20 \mathrm{~min}$ después de la administración del fármaco anestésico, se procedió vía ámpula rectal, a la estimulación digital de la próstata. Posteriormente el pene del animal fue expuesto y se comprobó la presencia de espículas peneanas. El pene fue lavado con solución fisiológica (cloruro de sodio, $0.9 \%$ ) y secado con una toalla de papel. Seguidamente, se insertó en la uretra del pene una sonda uretral (sin ventana lateral, $1 \mathrm{~mm}$ de diámetro y $13 \mathrm{~mm}$ de longitud) estéril y lubricada (Provar ${ }^{\circledR}$ Cata Ltda, São Paulo, Brasil) hasta una profundidad de 7 a $9 \mathrm{~cm}$. Después de unos segundos se acopló en la sonda una jeringa de $1.0 \mathrm{~mL}$ y se fue realizada una presión negativa para aumentar el efecto de succión. Una vez realizado lo anterior la sonda se retiró de la uretra (Fig. 1) y la muestra seminal fue colocada en un tubo de polipropileno de 1.5
$\mathrm{mL}$ precalentado, conteniendo $50 \mu \mathrm{L}$ de un diluyente a base de yema de huevo (Tris-huevo: $3.00 \%(\mathrm{p} / \mathrm{v})$ Tris (hidroximetil) amino (Synth, Brasil), $20.00 \%$ (v/v) de yema de huevo, 1.05 $\%(\mathrm{p} / \mathrm{v})$ de ácido cítrico (Synth, Brasil) 1.25 $\%(\mathrm{p} / \mathrm{v})$ de glucosa (VETEC , Brasil), $0.13 \%$ $(\mathrm{v} / \mathrm{v})$ de sulfato de gentamicina (Novafarma Productos farmacéuticos, Brasil) $0.50 \%(\mathrm{p} / \mathrm{v})$ de lauril sulfato de sodio (VETEC, Brasil) en agua destilada, $\mathrm{pH}$ 7.4). Una vez finalizados los procedimientos, se procedió a la administración del fármaco reversor del protocolo anestésico (Atipamezole $4.28 \mathrm{mg} / \mathrm{ml}$, Vetoquinol Orión Grupo Pharma Orion, Italia) en una dosis de $0.25 \mathrm{mg} / \mathrm{kg}$. El animal se trasladó al recinto donde fue monitoreada su recuperación post anestesia.

Análisis y criopreservación del esperma: Inmediatamente después de la recolecta seminal se evaluó la motilidad espermática, por medio de microscopio binocular, con un contraste de fases y un aumento de $100 \mathrm{X}$. Una vez confirmada la presencia de espermatozoides en la muestra, esta fue protegida de la temperatura y luz y se envió al sector RAOV-HOSPMEV / UFBA. Se cuantificó el volumen de la muestra con una micropipeta. El movimiento masal fue estimado basado en el movimiento de las células (Walton, 1952). El vigor espermático, el porcentaje de motilidad total (MT) y motilidad progresiva (MP) fueron estimadas subjetivamente por el mismo técnico en un microscopio binocular con contraste de fases $(100 \mathrm{X})$. La concentración espermática se estimó usando un hemocitómetro. La integridad estructural de la membrana (EOS) se
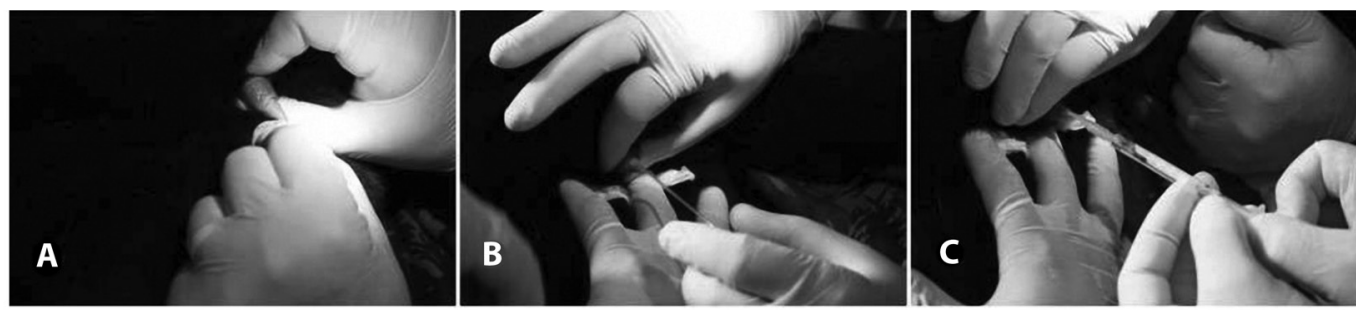

Fig. 1. Recolección de semen en el jaguar (Panthera onca). (A) Limpieza del pene; (B) Introducción de sonda uretral; (C) Aspiración del semen con uso de jeringa de $1 \mathrm{~mL}$ acoplado en la sonda. Parque Zoobotánico Getulio Vargas, Salvador-Bahia, Brasil. 
evaluó con la prueba supra vital utilizando el colorante eosina en un microscopio de luz con un aumento de 400 X (Cedenho \& Tokunaga, 1995). La integridad funcional de la membrana plasmática $(\mathrm{CH})$ se estimó mediante una prueba hipo osmótica (Comercio, Monachesi, Loza, Gambarotta, \& Wanke, 2013; Menezes et al., 2013). La morfología espermática se evaluó con una preparación de solución salina tamponada de formaldehído (Hancock, 1956). Las anomalías morfológicas fueron clasificadas en células con defectos menores y mayores (Blom, 1973). Con los parámetros de motilidad total y progresiva se estimó el índice de motilidad espermática (SMI) utilizando la ecuación $S M I=\frac{M T+\left(M P P_{2} 20\right)}{2}$ (Baudi, et al., 2008; Morais, Mucciolo, Gomes, \& Lacerda, 2002; Morato, et al., 2001; Morato, et al., 2004).

Posterior a la evaluación del semen precongelación, las muestras fueron diluidas con diferentes crio protectores experimentales, descritos a continuación: el crio protector no penetrante Tris-yema de huevo fue utilizado como base para la congelación de muestras, diferenciados por el crio protector penetrante grupo 1 (GLI) con $6 \%$ de glicerol (Cromaline química fina, Sao Paulo, Brasil); grupo 2 (DMF) con $3 \%$ de dimetilformamida (Cromaline química fina, Sao Paulo, Brasil) y grupo 3 (DMA) con $3 \%$ de NN- Dimetilacetamida (DMA) (Sigma-Aldrich, Sao Paulo, Brasil). Las muestras se envasaron en pajuelas de 0.25 $\mathrm{mL}$ y se colocaron horizontalmente en una plataforma de poliestireno de $5 \mathrm{~cm}$ de altura y fueron sometidas a enfriamiento $\left(0.47{ }^{\circ} \mathrm{C} /\right.$ min) a una temperatura de $5{ }^{\circ} \mathrm{C}$, con un tiempo de equilibrio de $120 \mathrm{~min}$. Posteriormente, la plataforma fue retirada de la refrigeración y se colocó en vapor de nitrógeno líquido a $5 \mathrm{~cm}$ de profundidad de la lámina líquida, durante 20 min (Paz, et al., 2000). Posterior a ello, las pajuelas fueron sumergidas en nitrógeno líquido a una temperatura de $-196{ }^{\circ} \mathrm{C}$ y se colocaron en raquetas identificadas y almacenadas en un tanque de nitrógeno hasta el momento de la descongelación.

Análisis de muestras congeladas-descongeladas: Las pajuelas fueron descongeladas a una temperatura de $37{ }^{\circ} \mathrm{C}$ durante $30 \mathrm{~s}$. Se evaluó subjetivamente la integridad estructural y funcional de la membrana plasmática y morfología espermática. Adicionalmente la muestra se sometió a un análisis seminal asistido por computadora (CASA) y al análisis de citometría de flujo. Para el análisis seminal asistido por computadora, se utilizó el sistema Sperm Class Analyser (SCA, Microptic, España). Los parámetros evaluados fueron: velocidad rectilínea $(\mu \mathrm{m} / \mathrm{s}, \mathrm{VSL})$, velocidad curvilínea $(\mu \mathrm{m} / \mathrm{s}, \mathrm{VCL})$, velocidad de trayectoria media $(\mu \mathrm{m} / \mathrm{s}$, VAP), amplitud del desplazamiento lateral de la cabeza $(\mu \mathrm{m}, \mathrm{ALH})$, frecuencia entrecruzamiento $(\mathrm{BCF}, \mathrm{Hz}), \mathrm{WOB}$ (VAP/VCL), índice de linealidad (\%, LIN) e índice de rectitud (STR \%).

La evaluación de la integridad de la membrana plasmática se realizó en el Laboratorio de Biología Molecular de la Fundación Oswaldo Cruz (Fiocruz, Bahia). Se utilizaron dos sondas fluorescentes: el diacetato de carboxifluoresceína (DIC, 21879, Sigma Aldrich), que emite fluorescencia verde y yoduro de propidio (PI, P4170, Sigma Aldrich), que emite fluorescencia de color rojo, en una solución de trabajo (Harrison \& Vickers, 1990; Mendoza, et al., 2012). Se añadió a cada muestra un volumen de $20 \mu \mathrm{L}$ de semen diluido en $150 \mu \mathrm{L}$ de PBS a una concentración de al menos $0.25 \times 10^{6}$ espermatozoides $/ \mathrm{mL}$ y $5 \mu \mathrm{L}$ de IP e $5 \mu \mathrm{L}$ de DIC en cada tubo criogénico. Las células espermáticas se clasificaron como membrana intacta (DIC + / IP -) y células con daños en la membrana (DIC + / IP +; DIC - / IP +; DIC - / $\mathrm{IP})$. Las muestras se analizaron con un citómetro de flujo con un espectro entre $250-850 \mathrm{~nm}$ (BD LDRFortessa, BD Bioscience, EE.UU.). Las imágenes se analizaron con el software FlowJo ${ }^{\circledR}$ (FlowJo LLC, EE.UU.)

Se realizaron pruebas de normalidad y homocedasticidad mediante las pruebas Shapiro-Wilk y Levene respectivamente. Posteriormente para comparar los métodos de medición testicular, se realizó una prueba " $t$ " para muestras independientes. Por otro lado, para la comparación de medias para las características del semen (Microscopía, CASA y citometría 
de flujo) se utilizó la prueba no paramétrica de Kruskal Walis. Se realizó la prueba de Wilcoxon para diferencias entre pares ordenados de los parámetros seminales, características de integridad de las membranas y el porcentaje de morfo anomalías antes y después de la congelación de cada muestra. Los análisis estadísticos fueron realizados en el programa SPSS versión 13.0.

\section{RESULTADOS}

Biometría: En la evaluación morfológica del sistema genital, se observó que el pene presenta un aspecto normal y una inserción posterior, y se verificó la presencia de espículas peneanas en los individuos, excepto en el $L$. wiedii cuya ausencia de espículas es inherente a la especie (Morais et al., 2002). Los valores correspondientes a la biometría testicular se presentan en la tabla 1. La comparación de los parámetros testiculares, área, volumen, peso e IG, no presentaron diferencias $(\mathrm{P}<0.05)$ entre los grupos, según el método de toma de las medidas (pie de rey y ultrasonido).

Parámetros seminales: Los parámetros de la evaluación seminal por microscopía convencional, evaluados pre y post congelación puede observarse en la tabla 2. La muestra recolectada del $P$. onca, sufrió una contaminación ambiental por lo que no fue posible estimar la concentración espermática ni someter la muestra a crio preservación.

En la evaluación de la morfología las tres especies presentaron los defectos mayores de cola fuertemente enrollada/doblada y alteraciones de la pieza intermedia. En el caso de $P$. onca se encontró un $1 \%$; en cada caso; pseudogota, gota citoplasmática proximal y anomalías teratogénicas (Fig. 2).

Al evaluar los parámetros seminales postcongelación según el crio protector utilizado para el proceso de congelación, no hubo diferencias $(\mathrm{P}>0.05)$ entre los grupos. En la comparación de los parámetros espermáticos de integridad de membranas y morfología, evaluados en las muestras frescas y en las muestras congeladas-descongeladas (Tabla 2), no se encontraron diferencias $(\mathrm{P}>0.05)$ para los parámetros antes y después del proceso de criopreservación.

Análisis CASA y citometría de flujo: $\mathrm{La}$ comparación de los parámetros espermáticos evaluados por el sistema CASA entre grupos separados por crio protector, indicó que no hubo diferencias $(\mathrm{P}>0.05)$ entre los parámetros seminales entre los grupos (Tabla 3 ). Los resultados de la citometría de flujo, indicaron que no hubo diferencias $(\mathrm{P}>0.05)$ por el crio protector (Tabla 4).

TABLA 1

Valores de peso corporal y parámetros testiculares de felinos silvestres sometidos a cateterismo uretral

\begin{tabular}{lccc}
\multicolumn{1}{c}{ Parámetro } & Leopardus wiedi & Puma yagouaroundi & Panthera onca \\
Peso corporal $(\mathrm{kg})$ & 3.95 & 6.30 & 56.55 \\
Área total pier $\left(\mathrm{cm}^{2}\right)$ & 2.23 & 3.34 & 10.18 \\
Área total US $\left(\mathrm{cm}^{2}\right)$ & 1.70 & 2.40 & 9.06 \\
Volumen total pier $\left(\mathrm{cm}^{3}\right)$ & 5.35 & 7.12 & 62.43 \\
Volumen total US $\left(\mathrm{cm}^{3}\right)$ & 4.30 & 5.88 & 51.35 \\
Peso total pier (g) & 4.50 & 6.15 & 53.72 \\
Peso total US (g) & 5.60 & 7.45 & 65.30 \\
IG Total pier (\%) & 0.14 & 0.12 & 0.11 \\
IG total US (\%) & 0.11 & 0.10 & 0.09 \\
\hline
\end{tabular}

1. Pier: medición estimada utilizando el instrumento pie de rey; US: medida estimada utilizando ultrasonido; IG: Índice Gonadosomático. 
TABLA 2

Valores de los parámetros seminales estimados por microscopía convencional pre y pos proceso congelación - descongelación de muestras de felinos silvestres

\begin{tabular}{|c|c|c|c|c|c|c|}
\hline \multirow[b]{2}{*}{ Parámetro } & \multicolumn{3}{|c|}{ Precongelación } & \multicolumn{3}{|c|}{ Poscongelación (valores medios) } \\
\hline & $\begin{array}{l}\text { Leopardus } \\
\quad \text { wiedii }\end{array}$ & $\begin{array}{c}\text { Puma } \\
\text { yagouaroundi }\end{array}$ & $\begin{array}{c}\text { Panthera } \\
\text { onca }\end{array}$ & $\begin{array}{l}\text { Leopardus } \\
\text { wiedii }\end{array}$ & $\begin{array}{c}\text { Puma } \\
\text { yagouaroundi }\end{array}$ & $\begin{array}{c}\text { Panthera } \\
\text { onca }\end{array}$ \\
\hline Volumen $(\mu 1)$ & 20.00 & 20.00 & 700.00 & -- & -- & -- \\
\hline Vigor (1-5) & 4.00 & 3.00 & 1.00 & 1.00 & 1.00 & $\ldots$ \\
\hline MT (\%) & 90.00 & 60.00 & 20.00 & 20.00 & 20.00 & $\ldots$ \\
\hline MP $(\%)$ & 80.00 & 50.00 & 5.00 & 10.00 & 10.00 & $\ldots$ \\
\hline SMI & 85.00 & 55.00 & 12.50 & 20.50 & 20.50 & $\ldots$ \\
\hline Concentración (x106/mL) & 120.00 & 570.00 & $\ldots$ & -- & -- & -- \\
\hline EOS $(\%)$ & 63.00 & 63.00 & 78.00 & 22.00 & 53.00 & $\ldots$ \\
\hline $\mathrm{CH}(\%)$ & 81.00 & 88.00 & 86.00 & 90.00 & 70.00 & $\ldots$ \\
\hline Anomalias esp. Totales (\%) & 7.00 & 31.00 & 26.00 & 30.00 & 54.00 & $\ldots$ \\
\hline Anom. esp. Menores (\%) & 7.00 & 26.00 & 7.00 & 9.00 & 15.00 & $\ldots$ \\
\hline Anom. esp. Mayores (\%) & 5.00 & 5.00 & 19.00 & 21.00 & 35.00 & $\ldots$ \\
\hline
\end{tabular}

MT: motilidad total; MP: motilidad progresiva; SMI: índice de motilidad espermática; EOS: porcentaje de espermatozoides con la integridad estructural de la membrana plasmática; $\mathrm{CH}$ : porcentaje de espermatozoides con integridad funcional de la membrana plasmática. Anom. anomalías, Esp: espermatozoides, ... Dato no obtenido, --: Dato obtenido solamente precongelación.

TABLA 3

Valores de los parámetros seminales obtenidos por un sistema computarizado de análisis seminal (CASA) para los felinos silvestres. Sector de Reproducción Animal y Obstetricia Veterinaria, Universidad Federal de Bahía

\begin{tabular}{lcc}
\multicolumn{2}{c}{ Parámetro } & \multicolumn{2}{c}{ Especie } \\
VCL $(\mu \mathrm{m} / \mathrm{s})$ & 38.57 & Puma yagouaroundi ${ }^{*}$ \\
VSL $(\mu \mathrm{m} / \mathrm{s})$ & 16.72 & 29.99 \\
$\operatorname{VAP}(\mu \mathrm{m} / \mathrm{s})$ & 23.46 & 10.05 \\
$\mathrm{ALH}(\mu \mathrm{m})$ & 1.94 & 14.48 \\
$\mathrm{BCF}(\mathrm{Hz})$ & 4.30 & 1.91 \\
LIN $(\%)$ & 36.36 & 1.80 \\
STR $(\%)$ & 59.44 & 34.67 \\
WOB $(\%)$ & 55.98 & 58.28 \\
\hline
\end{tabular}

*Mediana estadística; VCL: velocidad curvilínea; VSL: velocidad de línea recta; VAP: velocidad de trayectoria media; ALH: amplitud del desplazamiento lateral de la cabeza; BCF: frecuencia entrecruzamiento; LIN: índice de linealidad; STR: índice de rectitud; WOB: grado de oscilación de la cabeza durante el trayecto actual.

\section{DISCUSIÓN}

Los parámetros testiculares de los animales, en la $P$. onca se han reportado un volumen testicular de $44.4 \pm 2.0 \mathrm{~cm}^{3}$ (Morato et al., 2004), así como se ha reportado un intervalo para este parámetro de 41.4 a $52.4 \mathrm{~cm}^{3}$ (Morato et al., 2001), estos datos son inferiores a lo encontrado en nuestro estudio. Sin embargo, Paz et al. (2000) indica un valor promedio de $51.4 \pm 24 \mathrm{~cm}^{3}$ en los testículos medidos con pie de rey y un intervalo de $34-70 \mathrm{~cm}^{3}$, este rango incluye el volumen estimado por tanto el método de medición con pie de rey y ultrasonido (62.43 y $51.35 \mathrm{~cm}^{3}$ respectivamente). Respecto al peso testicular en el $P$. onca se ha informado 


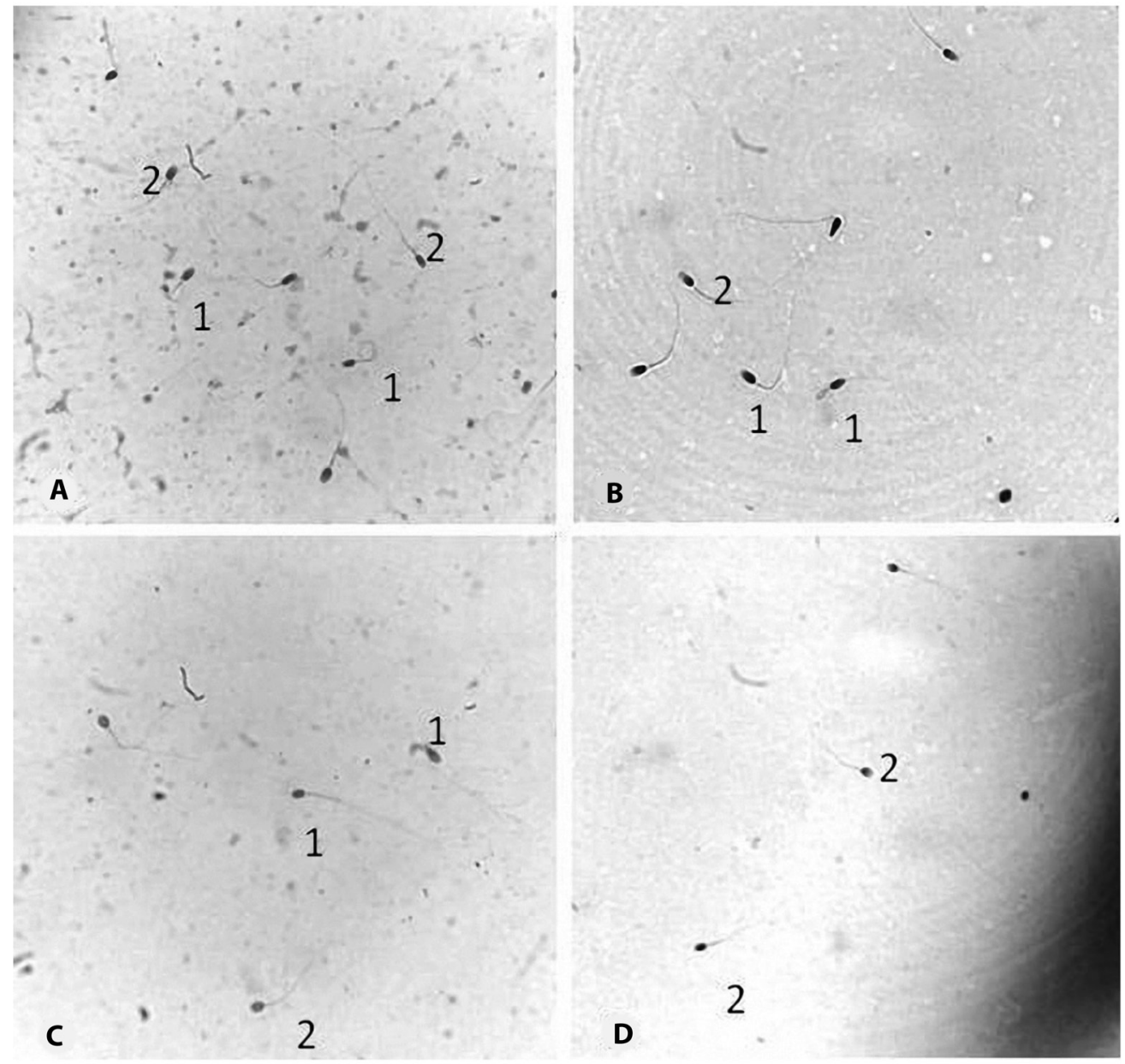

Fig. 2. Fotografía de espermatozoides a 1000 X (A) Puma yagouroundi, muestra evaluada in natura; (B) Puma yagouaroundi, muestra evaluada posterior a la congelación-descongelación (C) Leopardus wiedii, muestra evaluada in natura (D) Leopardus wiedii, muestra evaluada posterior a la congelación-descongelación. 1- Espermatozoides con morfo anomalías. 2- Espermatozoides con morfología normal. Sector de Reproducción Animal y Obstetricia Veterinari de HOSVET / UFBA, Salvador-Bahia, Brasil.

TABLA 4

Medianas estadísticas del parámetro de integridad de la membrana plasmática de las células de esperma felino criopreservado (GL, DMA, DMF) evaluado por citometría de flujo

\begin{tabular}{lcccccccc}
\multicolumn{1}{c}{ Especie } & DIC+ & IP+ & DIC- & IP- & DIC+IP- & DIC-IP+ & Esp. Memb. Íntegra & Esp. Memb. Dañada \\
Leopardus wiedi & 91.4 & 61.06 & 8.62 & 38.96 & 31.4 & 1.06 & 31.4 & 8.62 \\
Puma yaguaroundi & 91.78 & 88.84 & 8.22 & 11.6 & 7.88 & 4.15 & 15.18 & 8.22 \\
\hline
\end{tabular}

$\mathrm{DIC}+$ : cantidad de espermatozoides reactivos a diacetato de carboxifluoresceina; IP: cantidad de espermatozoides reactivos a yoduro de propídeo; DIC + | IP - : cantidad de espermatozoides con membrana plasmática intacta; DIC - | IP + : cantidad de espermatozoides con membrana plasmática dañada. 
un peso de $27.56 \pm 11.23 \mathrm{~g}$, no obstante, este parámetro presenta un alto coeficiente de variación entre los individuos $(\mathrm{r}=40.8)$.

Azevedo (2004) y Azevedo et al. (2010) indican la importancia de los parámetros de peso testicular en $P$. onca, ya que después de analizar los conductos seminíferos de los testículos de $P$. onca, encontró que estos animales tienen una reserva espermática de $165.9 \pm 22.3$ x $10^{6}$ espermatozoides por gramo de testículo. Vieira de Barros et al. (2017) observaron una correlación directa entre el peso testicular y la producción de espermática, debido a la correlación positiva entre el tamaño testicular y el tamaño del túbulo seminífero, de esta manera es posible por medio de las medidas testiculares estimar los componentes, longitud y reserva espermática en las gónadas de cada individuo.

En el $P$. yagouaroundi por su parte, solamente se ha reportado en la literatura un volumen testicular de $2.22 \mathrm{~cm}^{3}$ y un IG de $0.034 \%$ (Silva, 2014), estos datos son inferiores a los encontrados en este estudio. No obstante, debido a los escasos datos reportados para esta especie, se requiere de un mayor número de investigaciones para basar las comparaciones de los datos. Según Kenagy y Trombulak (1986) el tamaño de la masa testicular tiene relación con el peso corporal del animal, explicado por la formula donde la letra " $\mathrm{X}$ " representa el peso corporal, explicando la correlación positiva entre el peso corporal y el volumen del testículo. Esta correlación se demostró en este trabajo, donde el $P$. yagouaroundi, presentó un volumen testicular intermedio en comparación con un felino de porte grande ( $P$. onca) y un felino de porte pequeño ( $L$. wiedii). Afirmando que el volumen testicular es un parámetro relacionado con producción espermática.

Para felinos silvestres de pequeño porte, Morais et al. (2002) describe que el L. wiedii presenta parámetros testiculares y un peso corporal inferior al manigordo (L. pardalis) y superior al tigrillo (L. tigrinus). Para el manigordo se ha descrito un IG de $0.12 \pm 0.03 \%$ (Sarti, et al., 2009), entendiendo que el IG del manigordo debe ser ligeramente mayor que el IG estimado para el $L$. wiedii, esto es coincidente con el valor encontrado en este estudio (IG: 0.11). Adicionalmente, en el estudio de Sarti et al. (2009), el volumen testicular presentó una correlación alta y positiva con el peso corporal $(\mathrm{r}=0.9)$. Los autores mencionan que los datos biométricos de los felinos de pequeño porte adultos conducen a la predicción del estado de la madurez sexual de cada individuo.

Por otra parte, en trabajos donde se ha evaluado métodos para medición testicular en mamíferos silvestres, se han reportado valores similares entre las medidas obtenidas utilizando un pie de rey y las medidas obtenidos por medio del ultrasonido (Peixoto, et al., 2016). Estos resultados son coincidentes con los obtenidos en el presente estudio, lo que indica el uso potencial del pie de rey para estimar mediciones testiculares en felinos silvestres. No obstante, debe realizarse la aclaración que por medio del ultrasonido es posible una evaluación con mayor precisión del parénquima testicular.

La información sobre biometría testicular en el presente estudio es de gran importancia en la comprensión de la biología de la reproducción, en particular en relación con los aspectos del comportamiento de los sistemas de apareamiento e incluso contribuyen en el desarrollo de protocolos para la reproducción asistida en mamíferos silvestres, principalmente aquellos en peligro de extinción (Sarti, et al., 2009).

Adicionalmente entre los principales resultados de este estudio, se encuentra la recolecta seminal utilizando la técnica del cateterismo uretral en las tres especies, con especial énfasis en el $P$. yagouaroundi y el $L$. wiedii. Según nuestro conocimiento, este es el primer estudio documentado en donde se realiza recolección seminal, crio preservación y análisis seminal completo en Puma yagouaroundi.

La técnica de recolecta seminal por cateterismo uretral fue desarrollada inicialmente utilizando como modelo experimental al gato doméstico (Zambelli et al., 2008; Zambelli, Raccagni, Cunto, Andreani, \& Isani, 2010). La técnica proporciona una opción rápida en comparación con la técnica por vagina artificial (Buranaamnuay, 2017), menor costo económico en equipo y una menor contaminación 
con orina en comparación con la técnica de electroejaculación en felinos (Lueders, Luther, Scheepers, \& van der Horst, 2012; Pukazhenthi \& Wildt, 2004), adicionalmente el cateterismo uretral ofrece menores complicaciones para la obtención de la muestra en comparación con la recolecta seminal del epidídimo (Filliers, et al., 2010). De esta forma, el cateterismo uretral posibilita la obtención de un mayor número de eyaculados con un proceso simplificado.

Al evaluar la calidad del semen fresco, se constató que en $P$. onca el volumen de semen recolectado se encuentra dentro del intervalo reportado para esta especie en la recolecta por electroejaculación (211.2-11 $300 \mu \mathrm{L}$ ) (Morato et al., 2001, 2004; Paz et al., 2000). La amplia variación reportada para $P$. onca es coincidente con la variación reportada para grandes felinos, siendo que el león africano presenta variación en volumen seminal de 100-1 $000 \mu \mathrm{L}$ (Lueders et al., 2012). En concordancia Araujo et al. (2018) realizaron una recolección seminal por cateterismo uretral en $P$. onca en cautiverio con volúmenes similares.

La motilidad total y progresiva reportada para semen de $P$. onca por varios autores (Morato et al., 2001; Morato et al., 2004; Paz et al., 2000), indican valores promedio de 64-90\%, estas diferencias pueden deberse a una contaminación sufrida en el diluyente base, pero tal alteración fue corregida tras la observación del semen en el microscopio, para la realización de la recolecta en las otras especies. Comparando los datos de otros estudios citados anteriormente, Paz et al. (2000) indicaron los valores de motilidad total en el rango de 10-77 $\%$. Así mismo los autores antes mencionados indican que el vigor espermático en muestras seminales de $P$. onca oscila entre 1-4, datos coincidentes con los parámetros encontrados en el presente estudio.

Por otro lado, al evaluar la morfología espermática, el porcentaje de espermatozoides normales fue un $74 \%$, siendo mayor que la media encontrada por Morato et al. (2004) y dentro del intervalo reportado para felinos silvestres de porte grande (Lueders et al., 2012).
La cantidad de defectos mayores precongelación (19\%) es inferior que lo reportado en animales de vida libre $(23.0 \pm 15.13 \%)$, sin embargo, es similar al reportado para animales en cautiverio $(21.0 \pm 6.6 \%)$ (Araujo et al., 2018). Por otra parte, el porcentaje promedio de defectos menores que se han reportado para semen de $P$. onca $(18.3 \pm 12.2 \%)$ es superior al porcentaje encontrado en ese estudio (7\%). $\mathrm{Al}$ igual que en el estudio realizado por Araujo et al. (2018), se encontraron alteraciones menores de cola doblada y enrollada, cabeza aislada normal y el defecto mayor de gota citoplasmática proximal.

Morato et al. (2001) indican que la morfología espermática se correlaciona positivamente con el volumen testicular $(\mathrm{r}=0.29, \mathrm{P}<0.05)$ y negativamente con el volumen del eyaculado $(\mathrm{r}=-0.34, \mathrm{P}<0.01)$ en el $P$. onca. Los mismos autores indican un porcentaje de espermatozoides. Lo que quiere decir que, al incrementar el porcentaje de espermatozoides morfo normales, se espera que el volumen del testículo sea mayor y el volumen del eyaculado sea menor, lo que observando los datos presentados por Morato et al. (2001) es coincidente con los resultados obtenidos en el presente trabajo.

Al evaluar la información de calidad seminal en los felinos de porte pequeño, se observa que el volumen del eyaculado obtenido por cateterismo uretral es inferior al volumen recolectado en felinos de similar porte por electroeyaculación (Angrimani, et al., 2017; Baudi et al., 2008; Morais et al., 2002), para L. wiedii $(500 \mu \mathrm{l})$ (Morais et al., 2002) y en felinos de similar porte como el manigordo y el tigrillo $(350-660 \mu 1)$. Sin embargo, el volumen es similar al obtenido en gatos domésticos sometidos a cateterismo uretral (Jelinkova, Vitasek, Novotny, \& Bartoskova, 2018; Zambelli et al., 2010).

La concentración espermática (esp./mL) para el L. wiedii (120 esp./mL) y el P. yagouaroundi (570 esp./mL) es superior a lo reportado para el $L$. wiedii pero se encuentra en el intervalo reportado para felinos de porte similar (Baudi et al., 2008; Morais et al., 2002), en relación con los parámetros de velocidad y movimiento, el \% MT $(90 \%)$ y el \% MP (80 
\%) del L.wiedii es superior a lo reportado para esta especie, y se encuentra por encima de lo reportado para otros felinos silvestres de porte pequeño (Morais et al., 2002). El P. yagouaroundi presentó un \% MT de $60 \%$ y un \% MP del $50 \%$, valor inferior a lo encontrado para los felinos silvestres de porte pequeño (Baudi et al., 2008; Morais et al., 2002). Las discordancias anteriores pueden deberse a la evaluación única de los ejemplares, en el que los estudios referenciados utilizan un mayor número de individuos y repeticiones.

Adicionalmente, el presente trabajo es el primer reporte de características seminales de movimiento y velocidad para $P$. yagouaroundi, por lo que no es posible realizar una comparativa con otras investigaciones.

En cuanto al análisis de morfología espermática, el porcentaje de espermatozoides con morfología normal que presentó el $L$. wiedii (93\%) se encuentra por encima de los parámetros estudiados en otros gatos silvestres de porte pequeño (Baudi et al., 2008; Morais et al., 2002). El P. yagouaroundi mostró valores dentro de los rangos reportados para la especie L. tigrinus $(59.2 \pm 3.5-76.8 \pm 0.9)$ (Baudi et al., 2008; Morais et al., 2002).

Adicionalmente, en estudios en muestras espermáticas de manigordo (Leopardus pardalis) se cuantificaron parámetros espermáticos superiores en comparación con el $L$. wiedii y Leopardus tigrinus (Morais et al., 2002) tomando como base los datos reportados por Morais et al. (2002), es coincidente que los parámetros seminales encontrados para el caucel, se encuentren por debajo de los datos reportados para el manigordo. Por otra parte, los datos seminales de este trabajo para el $L$. wiedii son superiores a lo encontrado para el tigrillo por el mismo autor.

Respecto a la crio preservación de espermatozoides, la carencia de diferencias estadísticas $(\mathrm{P}>0.05)$ indica que tanto el glicerol como los crio protectores penetrantes basados en amidas, preservan igualmente el esperma de los felinos silvestres. La calidad del seminal evaluada posterior a la crio preservación de los felinos de porte mediano y pequeño, indica que los parámetros del semen tienden a disminuir después de la crio preservación (Baudi et al., 2008). En nuestro estudio como era de esperar fue observado una disminución en el \% EOS y $\%$ HOST y en el porcentaje espermatozoides con morfología normal. No obstante, en la presente investigación no hubo diferencias estadísticas $(\mathrm{P}>0.05)$ entre los parámetros evaluados antes y después de la criopreservación.

Los parámetros seminales evaluados por el CASA, fueron inferiores a lo reportado para el gato doméstico (Filliers et al., 2010; Prochowska, Nizański, Ochota, \& Partyka, 2015; Prochowska, Niżański, \& Partyka, 2016). Estas diferencias pueden ser atribuidas además de la diferencia entre especies animales, a las diferencias de metodologías entre los estudios y el momento de evaluación de las características.

Respecto al análisis por medio de la citometría de flujo, en el semen de felinos silvestres, existe una carencia de literatura publicada, habiendo solamente de datos relacionados a felinos domésticos. En los estudios de Siemieniuch y Woclawek-Potocka (2008) y Prochowska et al. (2016) se utilizaron las sondas de yoduro de propidio (IP) asociada a la sonda SYBR-14 para determinar la peroxidación de la membrana plasmática en combinación con la sonda BODIPY correspondientemente. Por lo tanto, al evaluar los resultados de estos trabajos, se determinó que la cantidad relativa de células IP. Los datos presentados en este estudio mostraron un porcentaje inferior al número de células indicadas como IP- en los estudios anteriores.

Este estudio amplía conocimientos sobre la biotecnología reproductiva de las especies silvestres de felinos. Los beneficios en las técnicas de reproducción asistida y bancos de recursos genéticos para el manejo de la población, mejora la eficiencia en los programas de reproducción asistida, elimina el comportamiento de incompatibilidad entre animales, permite la reproducción de individuos genéticamente valiosos, previene la inseguridad del recurso genético en caso de desastres naturales y permite el intercambio genético entre poblaciones ex situ e in situ entre otras ventajas 
(Howard \& Wildt, 2009). No obstante, es necesario que los conocimientos sobre fisiología reproductiva y técnicas de reproducción asistida en animales silvestres indiquen un protocolo de contención farmacológica para cada especie, que incluya practicidad, eficiencia, seguridad, bajo costo y viabilidad de la recolecta (Juvenal, et al., 2008) combinado con métodos de almacenamiento del material seminal viables (Howard \& Wildt, 2009).

Declaración de ética: los autores declaran que todos están de acuerdo con esta publicación y que han hecho aportes que justifican su autoría; que no hay conflicto de interés de ningún tipo; y que han cumplido con todos los requisitos y procedimientos éticos y legales pertinentes. Todas las fuentes de financiamiento se detallan plena y claramente en la sección de agradecimientos. El respectivo documento legal firmado se encuentra en los archivos de la revista.

\section{AGRADECIMIENTOS}

Los autores agradecen al personal y las autoridades oficiales del Parque Zoobotánico Getulio Vargas, Salvador, Bahia, Brasil. A la Fundación de Apoyo a la Investigación del Estado de Bahía (FAPESB). Al Grupo Coimbra de Universidades Brasileñas (GCUB) y a la Organización de los Estados Americanos (OEA). El presente trabajo fue realizado con el apoyo de la CAPES, código de financiamiento 001 (O presente trabalho foi realizado com apoio da Coordenação de Aperfeiçoamento de Pessoal de Nível Superior - Brasil (CAPES) Código de Financiamento 001).

\section{RESUMEN}

Introducción: La administración de un fármaco agonista de un alfa- 2-adrenérgicos asociado con la técnica del cateterismo uretral, es un método de desarrollo reciente en la recolección seminal en felinos, ofreciendo las ventajas sobre la recolecta por electroeyaculación y la recolecta epididimaria. La técnica se ha probado en Panthera onca y felinos de pequeño porte. Objetivos: Realizar la recolección seminal en felinos neotropicales utilizando la técnica de cateterización uretral. Determinar los parámetros testiculares y seminales de muestras recolectadas por la técnica de cateterización uretral. Metodología: En este estudio se utilizó un individuo por especie de Panthera onca, Puma yagouaroundi y Leopardus wedii localizados en el parque Zoobotánico Getúlio Vargas, Salvador, Bahia, Brasil. El semen fue recolectado por medio del cateterismo uretral y se utilizaron los farmácos: clorhidrato de medetomidina $(0.1 \mathrm{mg} / \mathrm{kg})$ y ketamina $(5.0 \mathrm{mg} / \mathrm{kg})$. Durante el desarrollo del procedimiento, se realizó la recolecta de los datos biométricos corporales y testiculares, así como la ultrasonografía de los testículos. Mediante microscopia óptica, en las muestras seminales se determinó: el vigor espermático, motilidad total, motilidad progresiva, la integridad estructural y funcional de la membrana plasmática y la morfología espermática. Las muestras se sometieron al proceso de congelación - descongelación utilizando tres diferentes crioprotectores. Las muestras descongeladas fueron evaluadas por microscopía convencional, análisis seminal computarizado y citometría de flujo. Resultados: En este estudio fue realizado con éxito la técnica de recolección seminal en las tres especies, siendo el primer estudio que informa de los parámetros seminales de $P$. yagouaroundi y el primero que reporta la recolección seminal uretral en $L$. wiedii. Los datos presentados describen de forma pionera la morfocinética espermática de las especies incluidas. No hubo diferencias $(\mathrm{P}>0.05)$ en la calidad seminal evaluando por grupo de crioprotector, ni diferencias en la integridad de las membranas pré e post-congelamento. Fue posible solamente la comparación con otras técnicas de recolección seminal o con resultados de calidad seminal obtenidos de muestras de gato doméstico. Conclusión: La cateterización uretral asociada al fármaco alfa- 2-adrenérgicos es una técnica eficiente para la recolección seminal en felinos neotropicales. El establecimiento de una potencial asociación de las características físicas del animal y la calidad seminal, es información de suma importancia en la aplicabilidad para la elección de reproductores en los programas de reproducción asistida.

Palabras clave: recolecta seminal; Panthera onca; Leopardus wiedii; Puma yaguaroundi; citometría de flujo.

\section{REFERENCIAS}

Angrimani, D. S. R., Barros, P. M. H., Losano, J. D. A., Cortada, C. N. M., Bertolla, R. P., Guimarães, M. A. B. V., ... Nichi, M. (2017). Effect of different semen extenders for the storage of chilled sperm in Tigrina (Leopardus tigrinus). Theriogenology, 89(1), 146154. DOI: $10.1016 /$ j.theriogenology.2016.10.015

Araujo, G. R., Paula, T. A. R., Deco-Souza, T., Morato, R. G., Bergo, L. C. F., Silva, L. C., ... Braud, C. (2018). Comparison of semen samples collected from wild and captive $P$. onca (Panthera onca) by urethral catheterization after pharmacological induction. 
Animal Reproduction Science, 195(December 2017), 1-7. DOI: 10.1016/j.anireprosci.2017.12.019

Azevedo, M. H. F. (2004). Análise morfofuncional do testículo da onça pintada (Panthera onca) adulta (Tesis de maestría). Universidade Federal de Viçosa, Minas Gerais Brasil.

Azevedo, M. H. de, de Paula, T. A., Matta, S. L., Fonseca, C. C., da Costa, E. P., Costa, D. S., \& Peixoto, J. V. (2010). Cell population indexes of spermatogenic yield and testicular sperm reserves in adult jaguars (Panthera onca). Animal Reproduction Science, 118(1), 83-88. DOI: 10.1016/j. anireprosci.2009.05.018

Baudi, D. L. K., Jewgenow, K., Pukazhenthi, B. S., Spercoski, K. M., Santos, A. S., Reghelin, A. L., ... Morais, R. N. (2008). Influence of cooling rate on the ability of frozen-thawed sperm to bind to heterologous zona pellucida, as assessed by competitive in vitro binding assays in the ocelot (Leopardus pardalis) and tigrina (Leopardus tigrinus). Theriogenology, 69(2), 204211. DOI: 10.1016/j.theriogenology.2007.09.013

Blom, E. (1973). The ultrastructure of some characteristic sperm defects and a proposal for a new classification of the bull spermogram. Nordisk Veterinaermedicim, 25(7), 383-391.

Buranaamnuay, K. (2017). Protocols for sperm cryopreservation in the domestic cat: A review. Animal Reproduction Science, 183(November 2016), 56-65. DOI: 10.1016/j.anireprosci.2017.06.002

Cedenho, A., \& Tokunaga, I. (1995). Coloração supravital para espermatozoides: comparação entre as tecnicas de eosina e eosina-nigrosina. Journal Brasileiro de Urología, 16(4), 234-236.

CITES. (2017). CITES Appendixes. Retrieved from https:// cites.org/sites/default/files/eng/app/2017/E-Appendices-2017-10-04.pdf

Comercio, E. A., Monachesi, N. E., Loza, M. E., Gambarotta, M., \& Wanke, M. M. (2013). Hypo-osmotic test in cat spermatozoa. Andrologia, 45(5), 310-314. DOI: doi.org/10.1111/and.12007

Filliers, M., Rijsselaere, T., Bossaert, P., Zambelli, D., Anastasi, P., Hoogewijs, M., \& Van Soom, A. (2010). In vitro evaluation of fresh sperm quality in tomcats: A comparison of two collection techniques. Theriogenology, 74(1), 31-39. DOI: 10.1016/j. theriogenology.2009.12.016

Hancock, J. (1956). The morphology of boar spermatozoa. Journal of Microscopy, 76(3), 84-97.

Harrison, R. A. P., \& Vickers, S. E. (1990). Use of fluorescent probes to assess membrane integrity in mammalian spermatozoa. Journal Reproduction Fertility, 88(1), 343-352.
Howard, J. G., Brown, J. L., Bush, M., \& Wildt, D. E. (1990). Teratospermic and Normospermic Domestic Cats: Ejaculate Traits, Pituitary-Gonadal Hormones, and Improvement of Spermatozoal Motility and Morphology After Swim-Up Processing. Journal of Andrology, 11(3), 204-215. DOI: 10.1002/j.19394640.1990.tb03229.x

Howard, J. G., \& Wildt, D. E. (2009). Approaches and efficacy of artificial insemination in felids and mustelids. Theriogenology, 71(1), 130-148. DOI: 10.1016/j. theriogenology.2008.09.046

Jelinkova, K., Vitasek, R., Novotny, R., \& Bartoskova, A. (2018). A comparison of quality parameters of fresh feline ejaculates collected by three different collection techniques. Reproduction in Domestic Animals, 53(5), 1068-1074. DOI: 10.1111/rda.13205

Johnson, L., Petty, C. S., \& Neaves, W. B. (1981). A new approach to quantification of spermatogenesis and its application to germinal cell attrition during human spermiogenesis. Biology of Reproduction, 25(1), $217-$ 226. DOI: $10.1095 /$ biolreprod25.1.217

Juvenal, J., Erdmann, R., Moreira, N., Moraes, W., Cubas, P., Delgado, L. E., ... Pachaly, J. R. (2008). Contenção farmocolõgica do gato-do-mato-pequeno Leopardus tigrinus, para colheita de semen, pela associação de tiletamina zolazepam e xilazina. Pesquisa Veterinaria Brasileira, 11(28), 541-546.

Kenagy, G., \& Trombulak, S. C. (1986). Size and Function of Mammalian Testes in Relation to Body Size. Journal of Mammalogy, 67(1), 1-22. Retrieved from http://www.jstor.org/stable/1380997

Lueders, I., Luther, I., Scheepers, G., \& van der Horst, G. (2012). Improved semen collection method for wild felids: Urethral catheterization yields high sperm quality in African lions (Panthera leo). Theriogenology, 78(3), 696-701. DOI: 10.1016/j. theriogenology.2012.02.026

Menezes, G. F. O. de, Bittencourt, R. F., Ribeiro Filho, A. de L., Oba, E., Chalhoub, M., Bittencourt, M. F., \& Bicudo, S. D. (2013). Utilização do choque osmótico na avaliação da viabilidade de sêmen criopreservado de ovinos. Brazilian Journal of Veterinary Research and Animal Science, 50(5), 396-405. Retrieved from http://revistas.usp.br/bjvras/article/ view/79940/83880

Mendoza, N., Casao, A., del Valle, I., Serrano, E., Nicolau, S., Asumpcao, M., ... Perez-Pe, R. (2012). Quality characteristics and fertilizing ability of ram sperm subpopulations separated by partition in an aqueous two-phase system. Journal of Chromatography B, 880(1), 74-81.

Micheletti, T., Cubas, Z., \& Moraes, W. (2012). Reprodução assistida em felídeos selvagens-uma revisão. Revista Brasileira de Reprodução Animal, 35(4), 408-417. 
Morais, R. N., Mucciolo, R. G., Gomes, M. L. F., \& Lacerda, O. (2002). Seasonal analysis of semen characteristics, serun testosterone and fecal androgens in the ocelot (Leopardus pardalis), margay (L. wiedii) and tigrina (L. tigrinus). Theriogenology, 57(1), 2027-2041.

Morato, R. G., Conforti, V. A., Azevedo, F. C., Jacomo, A. T. A., Silveira, L., Sana, D., ... Barnabe, R. C. (2001). Comparative analyses of semen and endocrine characteristics of free-living versus captive jaguars (Panthera onca). Reproduction, 122(5), 745-751. DOI: $10.1530 /$ rep.0.1220745

Morato, R. G., Verreschi, I. T. N., Guimarães, M. A B. V., Cassaro, K., Pessuti, C., \& Barnabe, R. C. (2004). Seasonal variation in the endocrine-testicular function of captive jaguars (Panthera onca). Theriogenology, 61(7-8), 1273-1281. DOI: 10.1016/j. theriogenology.2003.07.011

Moreira, N. (2017). Técnicas reprodutivas para a conservação de felídeos silvestres. Revista Brasileira de Reprodução Animal, 41(1), 116-120.

Paz, R. C. R. da, Zuge, R. M., Barnabe, V. H., Morato, R. L. G., Felippe, P. A. N., \& Barnabe, R. C. (2000). Penetration assay of frozen jaguar (Panthera onca) sperm in heterologous oocytes. Brazilian Journal of Veterinary Research and Animal Science, 37(1-6), 462-466.

Peixoto, G. C. X., Silva, M. A., Lima, G. L., Campos, L. B., Paiva, A. L. C., Paula, V. V., ... Silva, A. R. (2016). Use of Non-invasive Methods for Evaluating the Testicular Biometry in Collared Peccaries (Pecari tajacu Linnaeus, 1758). Journal of Veterinary Medicine Series C: Anatomia Histologia Embryologia, 45(1), 60-66. DOI: 10.1111/ahe.12171

Prochowska, S., Nizański, W., Ochota, M., \& Partyka, A. (2015). Characteristics of urethral and epididymal semen collected from domestic cats-A retrospective study of 214 cases. Theriogenology, 84(9), 15651571. DOI: $10.1016 / \mathrm{j}$.theriogenology.2015.08.005

Prochowska, S., Niżański, W., \& Partyka, A. (2016). Comparative analysis of in vitro characteristics of fresh and frozen-thawed urethral and epididymal spermatozoa from cats (Felis domesticus). Theriogenology, 86(8), 2063-2072. DOI: 10.1016/j. theriogenology.2016.07.002

Pukazhenthi, B. S., \& Wildt, D. E. (2004). Which reproductive technologies are most relevant to studying, managing and conserving wildlife? Reproduction, Fertility, and Development, 16(1-2), 33-46. DOI: 10.10371/RD03076
Sarti, P., Paula, T. A. R. De, Matta, S. L. P. Da, Fonseca, C. C., Poli, G. O., Balarine, M. K., \& Mascarenhas, R. M. (2009). Parâmetros biométricos corporais e testiculares de Jaguatiricas (Leopardus pardalis ) adultas. Revista Ceres, 56(2), 161-165.

Siemieniuch, M. J., \& Woclawek-Potocka, I. (2008). Assessment of Selected Quality Parameters of Epididymal Cat (Felis catus s. Domestica, L. 1758) Sperm Using Flow Cytometry Method and Computer Assisted Sperm Analyser. Reproduction of Domestic Animals, 43(5), 633-37. DOI: 10.1111/j.1439-0531.2007.00958.x

Silva, V. H. (2014). Avaliação do processo espermatogenico de gatos-mouriscos (Puma yagouarundi, LACEPEDE, 1809) adultos (Tesis de maestría). Universidade Federal de Viçosa, Minas Gerais Brasil.

Swanson, W., Roth, T., Citinc, S., Kenny, D., \& Wildt, D. (1996). Comparative cryopreservation and functionality of spermatozoa from the normospermic jaguar (Panthera onca) and teratospermic cheetah (Acinonyx jubatus). Theriogenology, 45(1), 241-241.

Vieira de Barros, C., Martins Galvão, N., Laís Croce, S., Ferreira Teles, T. F., Amorim de Souza, T., Dias de Araújo, V., ... Angrimani, D. (2017). Infertility diagnosis in Jaguar (Panthera onca): case report. Brazilian Journal of Veterinary Research and Animal Science, 53(3), DOI: 10.11606/issn.1678-4456. bjvras.2016.111568

Villaverde, A. I. S. B., Fioratti, E. G., Ramos, R. S., Neves, R. C. F., Ferreira, J. C. P., Cardoso, G. S., ... M. D. Lopes. (2014). Blood and seminal plasma concentrations of selenium, zinc and testosterone and their relationship to sperm quality and testicular biometry in domestic cats. Animal Reproduction Science, 150(12), 50-55. DOI: 10.1016/j.anireprosci.2014.08.004

Walton, A. (1952). Flow orientation as a possible explanation of "Wave-motion" and 'Rheotaxis" of spermatozoa. Journal Experimental Biology, 29(1), 520-531.

Zambelli, D., Prati, F., Cunto, M., Iacono, E., \& Merlo, B. (2008). Quality and in vitro fertilizing ability of cryopreserved cat spermatozoa obtained by urethral catheterization after medetomidine administration. Theriogenology, 69(4), 485-490. DOI: 10.1016/j. theriogenology.2007.10.019

Zambelli, D., Raccagni, R., Cunto, M., Andreani, G., \& Isani, G. (2010). Sperm evaluation and biochemical characterization of cat seminal plasma collected by electroejaculation and urethral catheterization. Theriogenology, 74(8), 1396-1402. DOI: 10.1016/j. theriogenology.2010.06.011 